\section{New video will promote silver diamine fluoride to children}

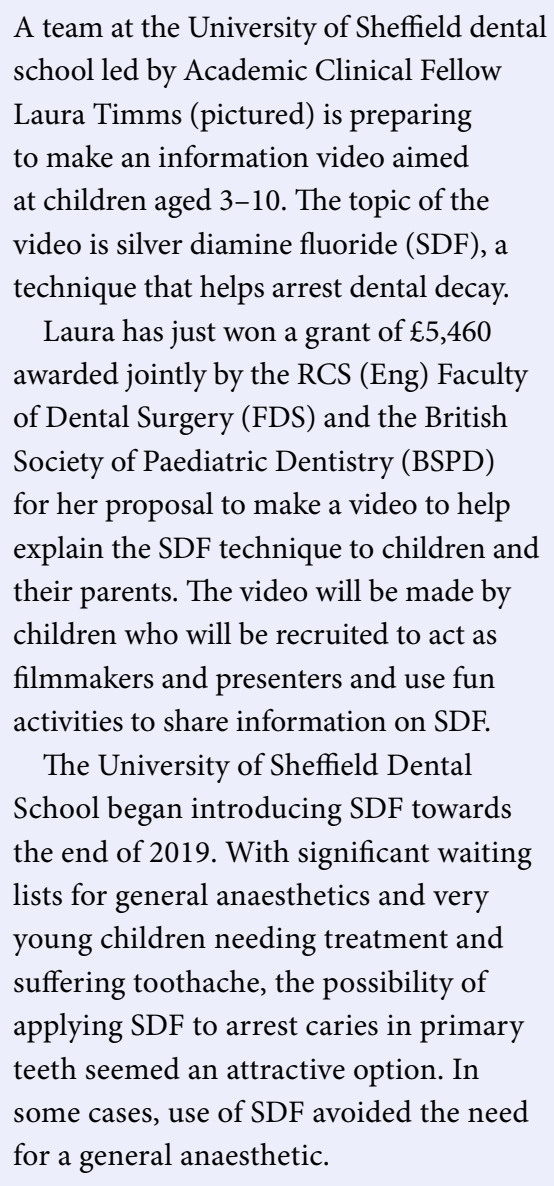

A team at the University of Sheffield dental school led by Academic Clinical Fellow Laura Timms (pictured) is preparing to make an information video aimed at children aged 3-10. The topic of the video is silver diamine fluoride (SDF), a technique that helps arrest dental decay.

Laura has just won a grant of $£ 5,460$ awarded jointly by the RCS (Eng) Faculty of Dental Surgery (FDS) and the British Society of Paediatric Dentistry (BSPD) for her proposal to make a video to help explain the SDF technique to children and their parents. The video will be made by children who will be recruited to act as filmmakers and presenters and use fun activities to share information on SDF.

The University of Sheffield Dental School began introducing SDF towards the end of 2019. With significant waiting lists for general anaesthetics and very young children needing treatment and suffering toothache, the possibility of applying SDF to arrest caries in primary teeth seemed an attractive option. In some cases, use of SDF avoided the need for a general anaesthetic.

SDF is widely used overseas and at the International Association of Paediatric Dentistry (IAPD) meeting in 2019, Laura attended sessions on the technique. She remembered the simplicity of application from a student elective trip in Cambodia in 2014 and considered how useful it could be for some of her patients. She decided to research SDF and with colleagues in Sheffield and Newcastle wrote a paper published in May this year, ${ }^{1}$ examining why SDF is not being used more widely in the UK.

SDF stains teeth black but in her experience, parents often welcome a treatment which stops the decay and may avoid extractions under general anaesthetic. Discussion with the child and parent is vital, she says.

Working with Connect Trainees Laura led a research project exploring paediatric dentists' attitudes to SDF and in May she delivered a webinar on the topic to the group. Soon afterwards, she heard that she had been awarded the RCS/BSPD research pumppriming grant for the educational video.

Laura said: 'I also want to get the views of dentists and dental therapists on SDF. It's an easy and simple treatment with the right

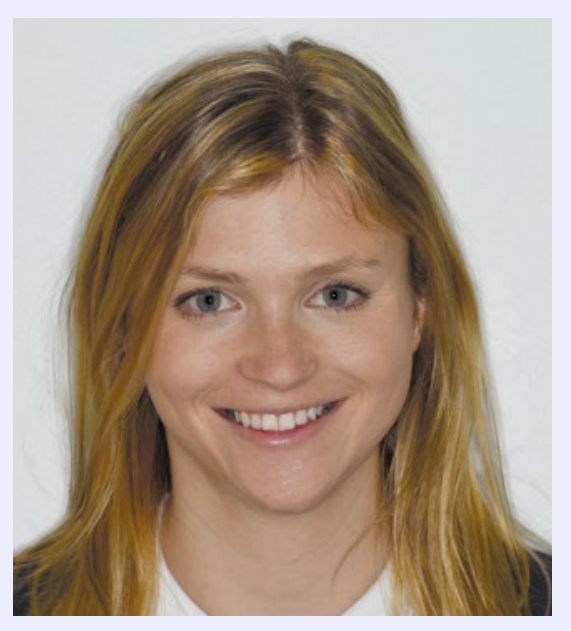

case selection and if it prevents children suffering with the pain of toothache and needing hospital treatment, it can only be a good thing.'

Sheffield-based Consultant in Paediatric Dentistry Professor Helen Rodd, one of Laura's supervisors, said that the impact of COVID-19 and the move to minimising aerosol generating procedures (AGPs) means that SDF is more valuable than ever for treating very young children and keeping them out of pain.

\section{Reference}

1. Timms L, Sumner O, Deery C, Rogers H J. Everyone else is using it, so why isn't the UK? Silver diamine fluoride for children and young people. Community Dent Health 2020; 37: 143-149.

\title{
Discovery could lead to stronger dental fillings
}

An international group of researchers led by Professor Owen Addison from King's College London has been able to close a gap in the knowledge of photo-activated resin-based composites, commonly used in medical and dental applications.

In a recent paper published in Nature Communications, the team from Canada, the United Kingdom, Norway and the United States described how they saw inside the resin matrix and gained insight into how filler particles interact with it during setting and influence the dental filling materials. ${ }^{1}$

Professor Addison said: 'We've been working for a number of years using synchrotron-based techniques primarily to try and understand the evolving structure of the polymer network of the resin matrix that makes up these materials.'

As the Chair of Oral Rehabilitation of King's College London's Faculty of
Dentistry, Oral \& Craniofacial Sciences and Adjunct Professor of Dentistry at the University of Alberta, Canada, Professor Addison has been interested in understanding the structure of these materials in order to improve their performance.

The team used a synchrotron technique that allows them to look at different resin chemistries and filler compositions. This will help researchers optimise the material, make it more resistant to wear and mechanical deterioration, and lead to less time spent in the dental clinic.

The technique, wide field mid-infrared imaging, was used at the Mid-IR beamline (https://bit.ly/2MVSsgP) at the Canadian Light Source (CLS) - a national research facility producing the brightest light in Canada - and allowed the team to gain a greater understanding of what was happening within the matrix of the resin composite. The filler particles are introduced to the material for better mechanical performance, but there has been a significant gap in the knowledge about the way these fillers affect the polymerisation, or hardening, of the material.

For the first time, the team was able to demonstrate that the fillers themselves modify the local reaction of the setting material. Professor Addison believes that this information has the potential to improve these resin composites, which would not only be an asset to dentistry but to other medical and industrial applications of photo-polymer composites.

\section{Reference}

1. Sirovica S, Solheim J H, Skoda M W A et al. Origin of micro-scale heterogeneity in polymerisation of photo-activated resin composites. Nat Commun 2020; 11: 1849. 